\title{
Celastrus orbiculatus extract triggers apoptosis and autophagy via PI3K/Akt/mTOR inhibition in human colorectal cancer cells
}

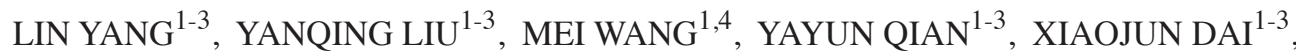 \\ YAODONG ZHU ${ }^{1-3}$, JUE CHEN ${ }^{1-3}$, SHIYU GUO ${ }^{5}$ and TADASHI HISAMITSU ${ }^{5}$
}

\begin{abstract}
${ }^{1}$ Institute of Traditional Chinese Medicine and Western Medicine, School of Medicine, Yangzhou University, Yangzhou, Jiangsu 225009; ${ }^{2}$ Jiangsu Key Laboratory of Integrated Traditional Chinese and Western Medicine for Prevention and Treatment of Senile Diseases, Yangzhou, Jiangsu 225001; ${ }^{3}$ Jiangsu Co-innovation Center for Prevention and Control of Important Animal Infectious Diseases and Zoonoses, Yangzhou, Jiangsu 225009; ${ }^{4}$ Department of Gastroenterology, The First People's Hospital of Yangzhou, The Second Clinical School of Yangzhou University, Yangzhou, Jiangsu 225000, P.R. China; ${ }^{5}$ Department of Physiology, School of Medicine, Showa University, Tokyo 142-8555, Japan
\end{abstract}

Received May 29, 2015; Accepted September 6, 2016

DOI: $10.3892 / 01.2016 .5213$

\begin{abstract}
Celastrus orbiculatus is used as a folk medicine in China for the treatment of numerous diseases. The ethyl acetate extract of Celastrus orbiculatus (COE) also displays a wide range of anti-cancer activities in the laboratory. However, the effectiveness of COE-induced autophagy and its mechanism of action in colorectal cancer cells have not been investigated thus far. The present study analyzed the effect of COE on HT-29 cell viability, apoptosis and autophagy using MTT assay, flow cytometry, transmission electron microscopy and western blotting. Additionally, the autophagy inhibitor 3-methyladenine and the autophagy inducer rapamycin were used to further explore the effects of COE-induced autophagy in HT-29 cells. The present study also examined whether the phosphatidylinositol-4,5-bisphosphate 3-kinase (PI3K)/Akt/mechanistic target of rapamycin (mTOR)/p70 ribosomal protein S6 kinase (p70S6K) signaling pathway was involved in the regulation of COE-induced autophagy. The results revealed that $\mathrm{COE}$ inhibited HT-29 cell proliferation and decreased cell survival in a time- and dose-dependent manner, and that COE possessed the ability to induce both apoptosis and autophagy in HT-29 cells. Furthermore, autophagy and apoptosis induced by COE synergized to inhibit colorectal cancer growth. In addition, COE treatment decreased the phosphorylation of Akt and its downstream effectors mTOR and p70S6K. Taken together, these results demonstrate that both autophagy and apoptosis were activated during COE treatment
\end{abstract}

Correspondence to: Professor Yanqing Liu, Institute of Traditional Chinese Medicine and Western Medicine, School of Medicine, Yangzhou University, 11 Huaihai Road, Yangzhou, Jiangsu 225009, P.R. China

E-mail: liuyanqing2014@163.com

Key words: Celastrus orbiculatus extract, autophagy, apoptosis of HT-29 cells, and that COE-induced autophagy decreases the viability of HT-29 cells via a mechanism that may depend on the PI3K/Akt/mTOR/p70S6K signaling pathway. Furthermore, compounds that induce autophagy administered in combination with COE may be an attractive strategy for enhancing the anti-tumor potency of COE in colorectal cancer.

\section{Introduction}

Celastrus orbiculatus belongs to the family Celastraceae and the genus Celastrus (1). The stem, roots and leaves of this plant are used as a folk medicine to treat rheumatoid arthritis (2). Celastrus and various of its defined constituents possess anti-cancer, anti-inflammatory and anti-oxidant properties (1-8). Our previous studies demonstrated that the ethyl acetate extract of Celastrus orbiculatus (COE) has significant anti-tumor effects in vitro and in vivo $(1,3,4,9-12)$. COE induced cytotoxicity and promoted the apoptosis of human hepatocellular carcinoma cells by inhibiting the Akt signaling pathway (9), and inhibited tumor angiogenesis by modulating the vascular endothelial growth factor signaling pathway (10). COE reduced the invasion and migration of gastric adenocarcinoma MGC-803 cells by reducing the expression and enzymatic activity of matrix metallopeptidase-9, which was regulated by inactivation of the phosphatidylinositol-4,5-bisphosphate 3-kinase (PI3K)/Akt signaling pathway and nuclear factor- $\kappa \mathrm{B}$ activity (11). COE also displayed anti-metastatic activity on human gastric adenocarcinoma by inhibiting the epithelial-mesenchymal transition (12). In previous experiments, the present authors also observed that COE induced autophagy and apoptosis in HT-29 cells; however, the effectiveness of COE induced-autophagy and its cellular mechanism in colorectal cells is still unknown.

Cell death can occur by apoptosis [also known as programmed cell death (PCD) I] or by necrosis and autophagy (which is known as PCD II) $(13,14)$. Drugs that induce apoptosis remain the most common chemotherapeutic agents used in medical oncology (13). Cancer cells hijack cell processes to survive, allowing them to escape from apoptosis and to acquire 
drug resistance (13). Drugs having both apoptotic and autophagic activities offer an added advantage to overcome these escape mechanisms. Autophagy is normally a degradative mechanism for the removal and turnover of bulk cytoplasmic constituents through the endosomal/lysosomal system (14-16); however, it is also an adaptive response to environmental changes, including nutrient deprivation, hypoxia and cell injury (17-19). Previous studies revealed that autophagy was involved in the cell death induced by anti-cancer drugs such as 5-fluorouracil and rapamycin $(20,21)$, and that autophagy may improve the efficacy of chemotherapy by enhancing the apoptosis of cells (22-24). By contrast, it has also been shown that autophagy is important in promoting cell survival against apoptosis (25-28). Despite this dual role of autophagy, the tissue/cancer-specific role of autophagy should be further explored to determine its potential as a therapeutic strategy for cancer and other human diseases. Thus, the aim of the present study was to investigate the effect of COE on autophagy and apoptotic cell death mechanisms, as well as their possible association in HT-29 cells treated with COE.

\section{Materials and methods}

Plant material. The stems of $C$. orbiculatus plants (production batch no. 070510) were purchased from Zhixin Pharmaceutical Co., Ltd. (Guangzhou, China). The COE was prepared and characterized at the Department of Chinese Materia Medica Analysis, China Pharmaceutical University (Nanjing, China). The chemical constituents and preparation procedure of COE were described previously (29,30). Prior to being used to treat cells, COE was dissolved in dimethyl sulfoxide (DMSO) and diluted to different concentrations. The final concentration of DMSO in the cell medium did not exceed $0.1 \%$.

Chemical reagents and antibodies. Antibodies against Beclin 1 (cat. no. 3495), light chain (LC) 3 (cat. no. 12741), B-cell lymphoma (Bcl)-2 (cat. no. 2872) and Bcl-2-associated X (Bax; cat. no. 2772), Akt (cat. no. 4691), Akt-Ser308 (cat. no. 9275), mechanistic target of rapamycin (mTOR; cat. no. 2983), mTOR-Ser2448 (cat. no. 5536), p70 ribosomal protein S6 kinase (p70S6K; cat. no. 2708) and p70S6K-Thr389 (cat.no. 9234) were purchased from Cell Signaling Technology, Inc. (Danvers, MA, USA). The enhanced chemiluminescence (ECL) kit was purchased from GE Healthcare Life Sciences (Chalfont, UK). DMSO and MTT were purchased from Sigma-Aldrich (Merck Millipore, Darmstadt, Germany). Other reagents were purchased from Beyotime Institute of Biotechnology (Jiangsu, China).

Cell culture. The human colorectal cancer cell line HT-29 was acquired from the Cell Bank of Chinese Academy of Sciences, Shanghai Institute of Cell Biology (Shanghai, China). HT-29 cells were cultured in RPMI-1640 medium (Gibco; Thermo Fisher Scientific, Inc., Waltham, MA, USA) containing 10\% fetal bovine serum (Gibco; Thermo Fisher Scientific, Inc.) and maintained at $37^{\circ} \mathrm{C}$ in a humidified incubator in an atmosphere of $5 \% \mathrm{CO}_{2}$.

Cell viability assay. Cell viability was measured by MTT assay. HT-29 cells were grown until logarithmic phase, and then seeded in a 96-well plate at a density of $1 \times 10^{4}$ cells/well overnight prior to treatment. The cells were then incubated with different concentrations of $\operatorname{COE}(0,20,40,80,160$ and $320 \mathrm{mg} / \mathrm{l})$ in triplicate. After 24, 48 and $72 \mathrm{~h}$ of incubation, the cells were incubated with medium containing MTT for $4 \mathrm{~h}$, and the formazan crystals were dissolved with $150 \mu \mathrm{l}$ DMSO. The plates were incubated on an agitator for $15 \mathrm{~min}$ at room temperature, and the absorbance was measured at $490 \mathrm{~nm}$ with a microplate reader. The drug concentration at which the cell viability was reduced to $50 \%$ [defined as the half maximal inhibitory concentration $\left.\left(\mathrm{IC}_{50}\right)\right]$ by $24 \mathrm{~h}$ of treatment was then determined. The effects of COE on cell viability were also determined in the absence or presence of the autophagy inhibitor 3-methyladenine (3-MA) (5 mM; Sigma-Aldrich; Merck Millipore) $3 \mathrm{~h}$ prior to COE treatment or the autophagy inducer rapamycin (100 nM; Sigma-Aldrich; Merck Millipore) $1 \mathrm{~h}$ prior to $\mathrm{COE}$ treatment to investigate the effects of COE-induced autophagy on the viability of HT-29 cells.

Annexin V-fluorescein isothiocyanate (FITC)/propidium iodide (PI) dual staining assay. Apoptosis was detected with the Annexin V-FITC/PI Apoptosis Detection kit (Nanjing KeyGen Biotech Co., Ltd., Nanjing, China). In brief, after 24 h of treatment with different concentrations of COE, cells were washed twice with ice-cold PBS and re-suspended in $500 \mu \mathrm{l}$ binding buffer. A total of $5 \mu \mathrm{l}$ annexin V-FITC and $5 \mu \mathrm{l}$ PI were added to the cell suspension and then incubated for $15 \mathrm{~min}$ at room temperature in the dark. FITC and PI fluorescence was analyzed in a FACSort flow cytometer (BD Biosciences, Franklin Lakes, NJ, USA) with CellQuest Pro software (BD Biosciences). The results were generated from three independent experiments.

Reverse transcription-quantitative polymerase chain reaction (RT-qPCR). Following treatment with COE, total RNA was extracted from the cells using the TRIzol reagent (Invitrogen; Thermo Fisher Scientific, Inc.) under RNase-free conditions, and RT was performed in a $20-\mu 1$ reaction with $200 \mathrm{ng}$ total RNA using the Two-Step RT-PCR kit (Takara Biotechnology Co., Ltd., Dalian, China). RT-qPCR was performed on a 7500 Real-Time PCR System (Applied Biosystems; Thermo Fisher Scientific, Inc.) using the SYBR Premix Ex Taq kit (Takara Biotechnology Co., Ltd.) in Axygen ${ }^{\circledR}$ 96-well reaction plates (Thermo Fisher Scientific, Inc.). PCR was performed under the following conditions: Initiation step of $95^{\circ} \mathrm{C}$ for $10 \mathrm{~min}$, denaturation step of $95^{\circ} \mathrm{C}$ for $15 \mathrm{sec}$, and the annealing and extension step at $60^{\circ} \mathrm{C}$ for $60 \mathrm{sec}$ for 40 cycles.

Primers were obtained from Shanghai Shenggong Biology Engineering Technology Service, Ltd. (Shanghai, China), and their sequences were: Beclin 1 (NM_003093), forward primer 5'-GGCTGAGAGACTGGATCAGG-3' and reverse primer 5'-CTGCGTCTGGGCATAACG-3'; LC3-II (NM_003094), forward primer 5'-GAGAAGCAGCTTCCTGTTCTGG-3' and reverse primer 5'-GTGTCCGTTCACCAACAGGAAG-3'; and GAPDH (NM_002046), forward primer 5'-TGGCACCCA GCACAATGAA-3' and reverse primer 5'-CTAAGTCATAGT CCGCCTAGA-3'. GAPDH was used as an internal control, and the data were analyzed using the $2^{-\Delta \Delta \mathrm{Cq}}$ method (31).

Ultrastructures observed by transmission electron microscopy (TEM). Following treatment with COE, the cells were 
washed with PBS, collected by centrifugation at $1,500 \times \mathrm{x}$ for 5 min at $4^{\circ} \mathrm{C}$, and fixed in $2.5 \%$ electron microscopy-grade glutaraldehyde (SenBeiJia Biological Technology Co. Ltd., Nanjing, China). The specimens were subsequently rinsed with $0.1 \mathrm{M}$ PBS, fixed in $1 \%$ osmium tetroxide (Nanjing Zhongjingkeyi Technology Co., Ltd., Nanjing, China), dehydrated through a graded series of ethanol and processed for Epon ${ }^{\mathrm{TM}}$ embedding (Sigma-Aldrich; Merck Millipore). Ultra-thin sections $(60 \mathrm{~nm})$ stained with uranyl acetate and lead citrate (Nanjing Zhongjingkeyi Technology Co., Ltd.) were observed with a JEM-1230 electron microscope (JEOL, Ltd., Tokyo, Japan).

Detection of acidic vascular organelles (AVOs) with acridine orange. To detect the AVOs in COE-treated cells in the presence of 3-MA and rapamycin, cells were stained with acridine orange (Beyotime Institute of Biotechnology). In acridine orange-stained cells, the cytoplasm fluoresces bright green, whereas the acidic compartments fluoresce bright red (20). The intensity of the red fluorescence is proportional to the degree of acidity and/or the volume of the cellular acidic compartment (20). Therefore, changes in the degree of acidity and/or the fractional volume of the cellular acidic compartments can be determined. Following treatment with COE for $24 \mathrm{~h}$, cells were fixed with chilled $70 \%$ ethanol and stained with $5 \mathrm{mM}$ acridine orange. Fluorescence was then observed using a fluorescence microscope (BX51; Olympus Corporation, Tokyo, Japan).

Western blot analysis. The expression levels of Beclin 1, LC3, Bax, Bcl-2 and mTOR-p70S6K signaling proteins (Akt, Akt-308, mTOR, mTOR-Ser2448, p70S6K and p70S6K-Thr389) in HT-29 cells were determined by western blot analysis. Briefly, a cell lysis solution was prepared using Cytoplasmic Extraction Reagent II (Fermentas; Thermo Fisher Scientific, Inc., Pittsburgh, PA, USA). A 50- $\mu \mathrm{g}$ sample of supernatant was subjected to $10 \%$ SDS-PAGE and then transferred onto nitrocellulose membranes (EMD Millipore, Billerica, MA, USA). The blot was blocked with $5 \%$ not-fat dry milk for $2 \mathrm{~h}$ at room temperature, and then incubated with primary antibodies $(1: 1,000)$ and agitated overnight at $4^{\circ} \mathrm{C}$. Subsequently, the membranes were washed three times with washing buffer for $10 \mathrm{~min}$ and incubated with secondary antibodies (1:1,000; G130321; Hangzhou Huaan Biotechnology Co., Ltd., Hangzhou, China) for $2 \mathrm{~h}$ at room temperature. $\beta$-actin (a housekeeping gene) was used as a loading control. ECL was used to detect the signals, using the SuperSignal West Pico Chemiluminescent Substrate (Thermo Fisher Scientific, Inc.) on a Molecular Imager ChemiDoc XRS System (Bio-Rad Laboratories, Inc., Hercules, CA, USA). Densitometry was determined using Quantity One 1-D analysis software (Bio-Rad Laboratories, Inc.).

Statistical analysis. Results are expressed as the mean \pm standard deviation. Each experiment was repeated $\geq 3$ times. Statistical comparisons of $\geq 2$ groups were conducted using analysis of variance followed by a Bonferroni post hoc test. All statistical analyses were performed using the SPSS 18.0 statistical software (SPSS, Inc., Chicago, IL, USA), and $\mathrm{P}<0.05$ was considered to indicate a statistically significant difference.

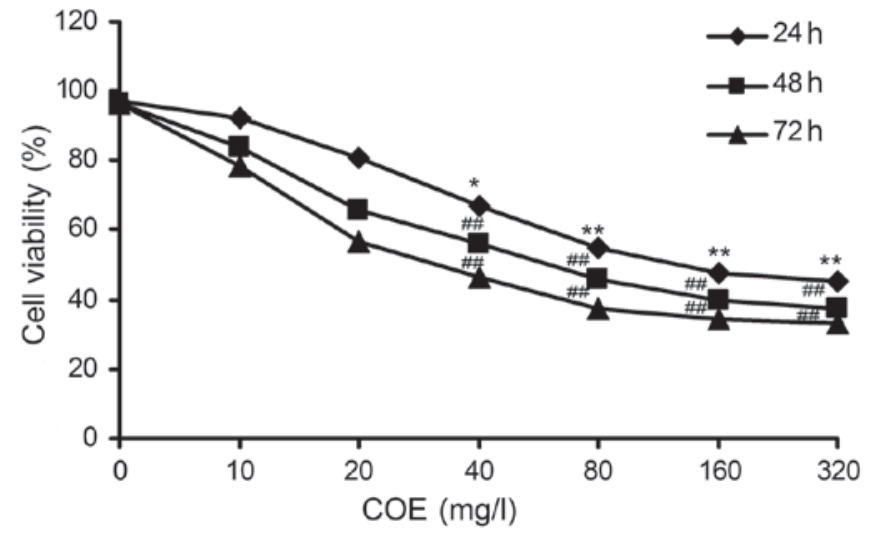

Figure 1. Reduction in HT-29 cell survival rates after the cells were treated with different concentrations of COE for varying lengths of time. Data are represented as the mean \pm standard deviation $(\mathrm{n}=3)$. ${ }^{*} \mathrm{P}<0.05$ and ${ }^{* *} \mathrm{P}<0.01$ compared with $0 \mathrm{mg} / 1$ COE treatment for $24 \mathrm{~h}$; ${ }^{\#} \mathrm{P}<0.05$ and ${ }^{\# \#} \mathrm{P}<0.01$ compared with $0 \mathrm{mg} / \mathrm{l} \mathrm{COE}$ treatment for 48 and $72 \mathrm{~h}$. COE, Celastrus orbiculatus extract.

\section{Results}

COE inhibits the proliferation of HT-29 cells. The results of the MTT assay revealed that HT-29 cell viability decreased in a dose-dependent manner upon administration of COE. A strong dose-dependent inhibition of cell proliferation was also observed in the HT-29 cell line. Upon treatment with $149.65 \pm 0.49 \mathrm{mg} / \mathrm{l}$ of COE for $24 \mathrm{~h}$, the cell viability was reduced to $50 \%$ (Fig. 1). These results indicate that $\mathrm{COE}$ exerts a potent anti-proliferative activity against colorectal cancer cells.

COE induces autophagy. The induction of autophagy is characterized by the formation of autophagosomes enclosed in a double membrane that fuse with lysosomes to form autolysosomes $(17,18)$. Electron microscopy observation of these cellular structures is the gold standard for detecting autophagy (22). In the present study, cells in the control group presented intact organelles. By contrast, large autophagosomes and autolysosomes were present in HT-29 cells treated with 80 or $160 \mathrm{mg} / \mathrm{l} \mathrm{COE}$ for $24 \mathrm{~h}$ (Fig. 2A).

Under normal conditions, the protein LC3 is dispersed throughout the cytoplasm in a dissociated form (LC3-I) (21). During autophagy induced by factors such as nutrient depletion, LC3-I is converted to its phosphatidylethanolamine-conjugated form (LC3-II), which localizes to both sides of the autophagosome (14). Beclin 1 is also indispensable for autophagy induction (13). The western blotting results revealed that the levels of LC3-II and Beclin 1 increased significantly in HT-29 cells treated with 80 or $160 \mathrm{mg} / \mathrm{l}$ COE for $24 \mathrm{~h}$ compared with control cells (Fig. 2B and C). No significant change was observed for LC3-II or Beclin 1 messenger RNA transcript levels.

COE induces apoptosis in colorectal cancer cells. As shown in Fig. 2D, ultrastructures in HT-29 cells were observed by TEM after $24 \mathrm{~h}$ of COE treatment at different concentrations. Cells in the control group exhibited intact organelles and round shape with nucleus and nucleolus chromatin. However, 
A

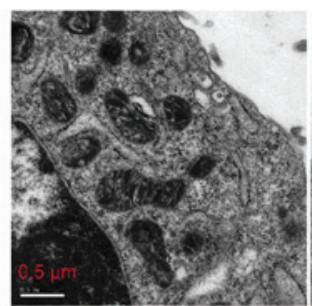

Control

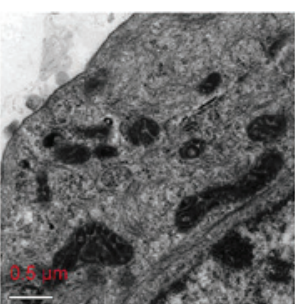

20

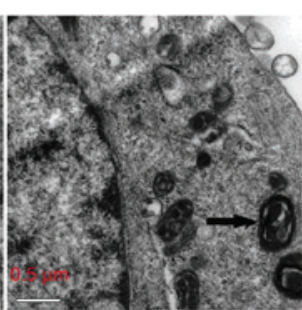

40

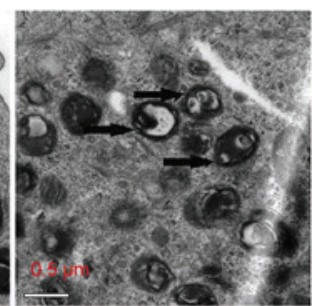

80

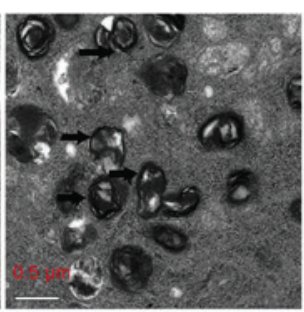

160
B

$$
\begin{array}{ccccc} 
& \multicolumn{3}{c}{\mathrm{COE}(\mathrm{mg} / \mathrm{l})} & \\
\cline { 2 - 5 } & & & &
\end{array}
$$

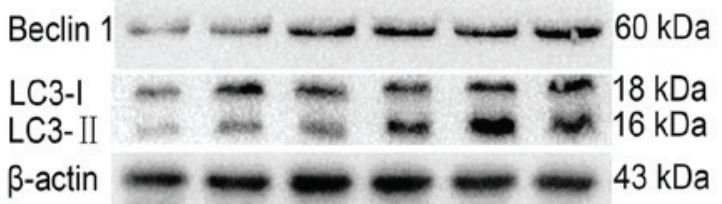

C

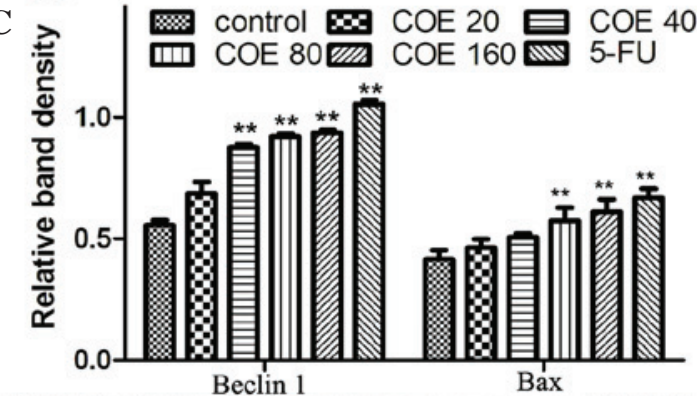

D

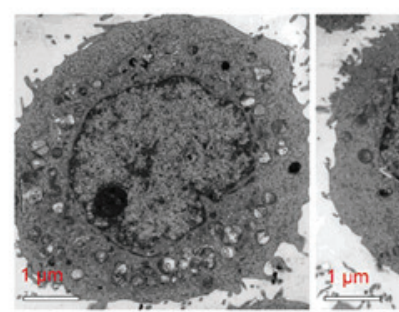

Control

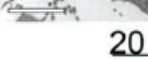

E
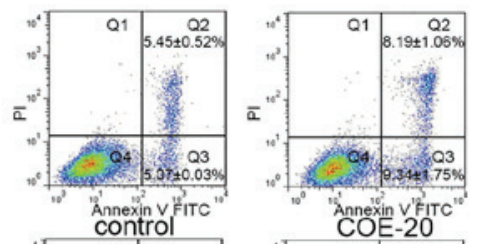

20

$\operatorname{COE}(\mathrm{mg} / \mathrm{l})$
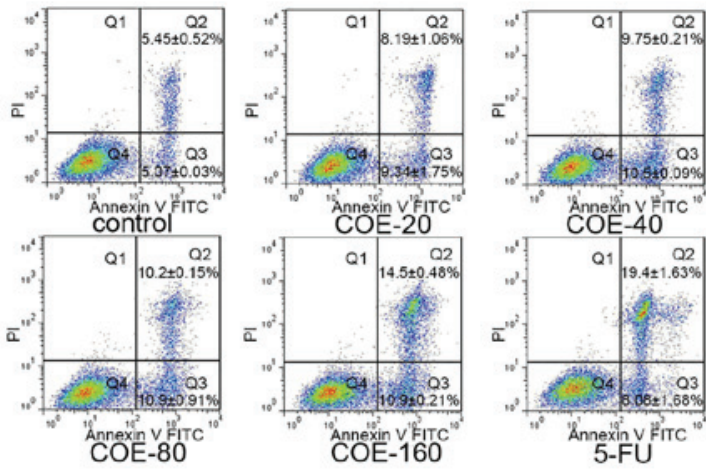

F

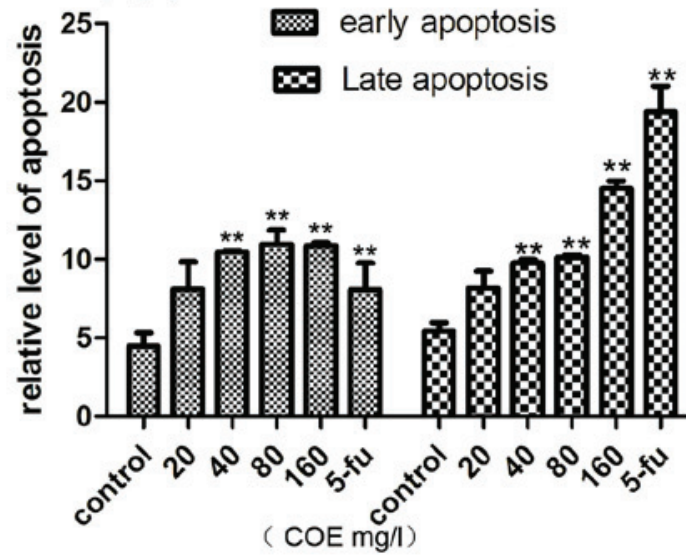

G
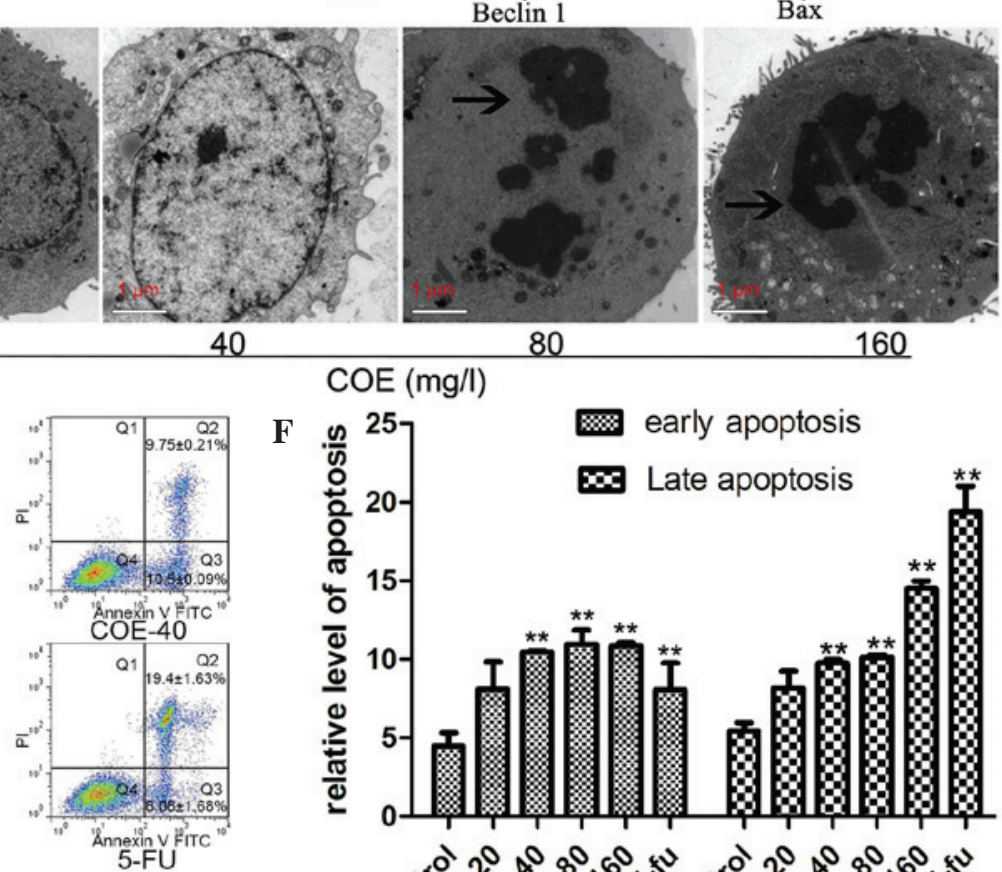

160

80

\section{.}

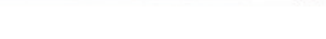




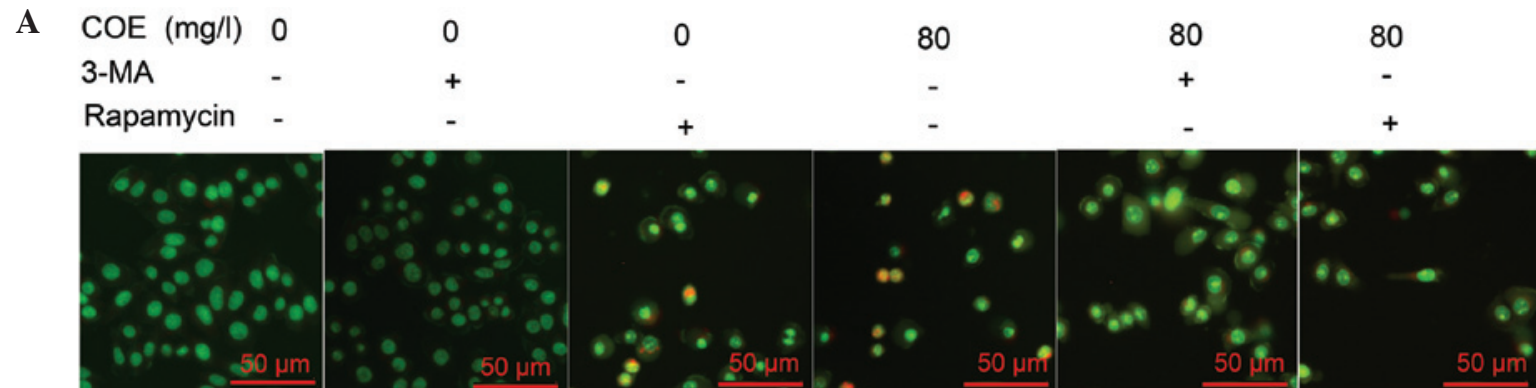

B

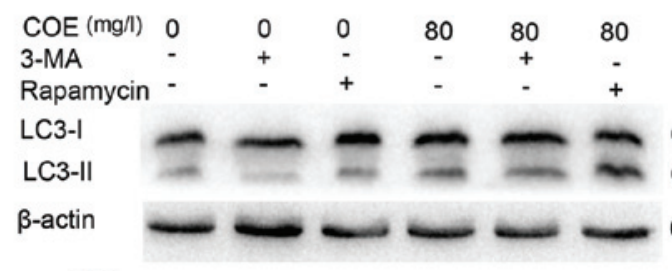

D

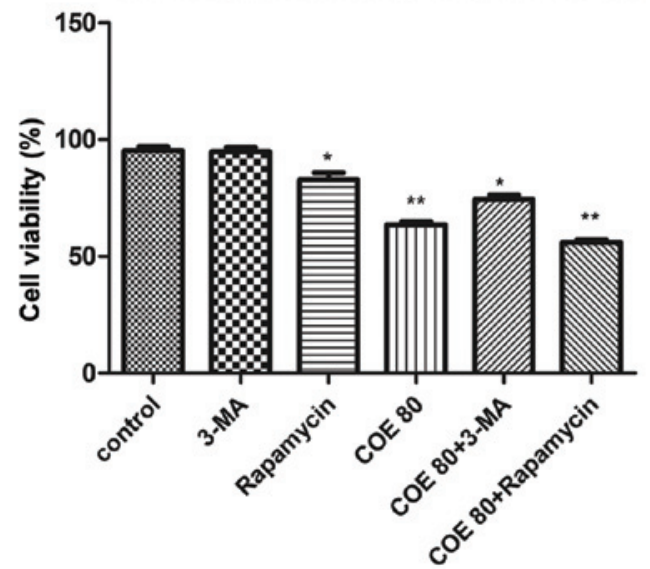

C

$(18 \mathrm{kDa})$

$(16 \mathrm{kDa})$

$(43 \mathrm{kDa})$

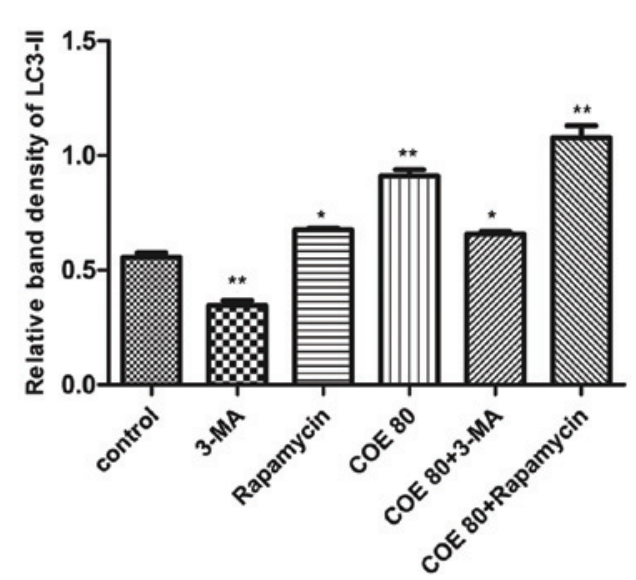

Figure 3. COE-induced cell death involves autophagy in HT-29 cells. (A) Detection of acidic vascular organelles in HT-29 cells treated with $80 \mathrm{mg} / \mathrm{l} \mathrm{COE} \mathrm{for}$ $24 \mathrm{~h}$ in the absence or presence of 3-MA or rapamycin by fluorescence microscopy with acridine orange staining. (B) Western blot analysis of the expression of LC3-II in HT-29 cells treated with $80 \mathrm{mg} / 1 \mathrm{COE}$ for $24 \mathrm{~h}$ in the absence or presence of 3-MA or rapamycin. (C) The band intensities of LC3-II were analyzed in HT-29 cells treated with $80 \mathrm{mg} / \mathrm{l} \mathrm{COE}$ for $24 \mathrm{~h}$ in the absence or presence of 3-MA or rapamycin. (D) Effect of $80 \mathrm{mg} / \mathrm{l} \mathrm{COE}$ treatment on the viability of HT-29 cells after $24 \mathrm{~h}$ in the absence or presence of 3-MA or rapamycin. ${ }^{*} \mathrm{P}<0.05$ and ${ }^{* *} \mathrm{P}<0.01$ compared with the untreated control. LC, light chain; COE, Celastrus orbiculatus extract; 3-MA, 3-methyladenine.

upon treatment with 80 or $160 \mathrm{mg} / \mathrm{l} \mathrm{COE}$, cell shrinkage, chromatin condensation and nuclear collapse were observed.

The percentage of total apoptotic cells was calculated by adding the percentages of early apoptotic-gated cells [annexin $\mathrm{V}^{+}$, quadrant (Q) 3 area] and late apoptotic-gated cells (annexin $\mathrm{V}^{+} / \mathrm{PI}^{+}, \mathrm{Q} 2$ area) (Fig. 2E). The results revealed a progressive increment in the cell population at the right-side coordinates upon COE administration in a dose-dependent manner compared with the control group (Fig. 2E and F).

Members of the Bcl-2 family serve a vital role in the regulation of apoptosis $(32,33)$. Thus, western blot analysis was used to determine the levels of Bax and Bcl-2. Compared with the control group, $\mathrm{Bcl}-2$ expression decreased with increasing concentrations of $\mathrm{COE}$, while Bax expression increased in a dose-dependent manner (Fig. 2G and H).

COE-induced cell death involves autophagy in HT-29 cells. Our data revealed that $<20 \mathrm{mg} / \mathrm{l} \mathrm{COE}$ had no significant impact on cell viability, while $80 \mathrm{mg} / \mathrm{l} \mathrm{COE}$ significantly inhibited HT-29 cell viability $(\mathrm{P}<0.01$; Fig. 1$)$. Based on the $\mathrm{IC}_{50}$ value, $80 \mathrm{mg} / \mathrm{l} \mathrm{COE}$ was selected explore the effects of COE-induced autophagy on HT-29 cell viability. To confirm whether
COE-induced autophagy was inhibited or enhanced by 3-MA or rapamycin, respectively, AVOs were observed by fluorescence microscopy. The results revealed that AVO formation induced by $80 \mathrm{mg} / \mathrm{l} \mathrm{COE}$ was reduced in the presence of 3-MA and increased in the presence of rapamycin (Fig. 3A). Additionally, western blot analysis demonstrated that the level of LC3-II was increased in the presence of rapamycin and decreased in the presence of 3-MA upon treatment with $80 \mathrm{mg} / \mathrm{l} \mathrm{COE}$ (Fig. 3B and C). In addition, to confirm whether COE-induced autophagy increased cell survival or cell death, a cell viability assay was performed following treatment of HT-29 cells with $80 \mathrm{mg} / \mathrm{l} \mathrm{COE}$ in the presence of 3-MA (an autophagy inhibitor) or rapamycin (an autophagy inducer) $(13,22)$. The ability of COE to inhibit proliferation was reduced in the presence of 3-MA and enhanced in the presence of rapamycin (Fig. 3D). These results indicate that COE-induced autophagy enhanced cell death rather than cell survival.

The PI3K-Akt-mTOR signaling pathway mediates COE cytotoxicity. To determine whether the PI3K-Akt-mTOR signaling pathway serves a role in mediating COE-induced apoptosis and autophagy, the levels of Akt, mTOR and p70S6K were 
A

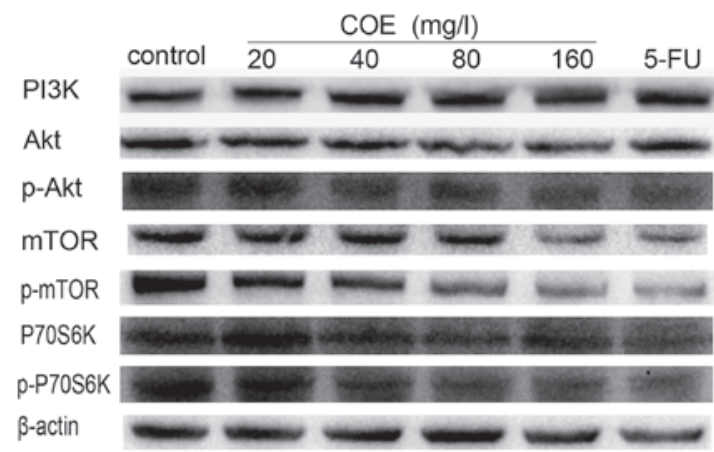

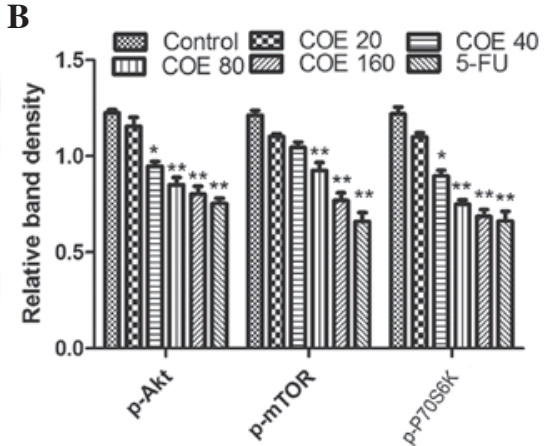

Figure 4. Protein expression of the PI3K/Akt/mTOR signaling pathway components in HT-29 cells treated with different concentrations of COE for 24 h. (A) The expression levels of PI3K, Akt, Akt-Ser308, mTOR, mTOR-Ser2448, p70S6K and p70S6K-Thr389 were analyzed by western blotting. (B) The band intensities of Akt-Ser308, mTOR-Ser2448 and p70S6K-Thr389 relative to the untreated control cells were determined upon normalizing to $\beta$-actin expression, and are expressed as the mean \pm standard deviation of three independent experiments. ${ }^{*} \mathrm{P}<0.05$ and ${ }^{* *} \mathrm{P}<0.01$ compared with the untreated control. $\mathrm{p}$, phosphorylated; FU, fluorouracil; PI3K, phosphatidylinositol-4,5-bisphosphate 3-kinase; mTOR, mechanistic target of rapamycin; p70S6K, p70 ribosomal protein S6 kinase; COE, Celastrus orbiculatus extract.

determined by western blotting. Following COE treatment, there was a robust and sustained decrease in Akt-Thr308, mTOR-Ser2448 and p70S6K-Thr389 levels in HT-29 cells (Fig. 4A and B). This indicated that COE may inhibit the proliferation of HT-29 cells by mediating the phosphorylation of Akt and reducing the kinase activity of mTOR. The reduction in mTOR activity promoted autophagy in HT-29 cells.

\section{Discussion}

As surgery and adjuvant chemotherapy are associated with low survival rates in colorectal carcinoma, there is an urgent requirement to identify novel therapeutic agents for this type of cancer (34). An ideal anti-cancer drug should initiate PCD in cancer cells, and consistent with this expectation, $\mathrm{COE}$ was observed to initiate this type of cell death $(25,34)$. In the current study, the anti-proliferative effects of COE were first examined. After incubation with different concentrations of COE for 24, 48 and $72 \mathrm{~h}$, the proliferation of HT-29 cells was significantly inhibited in a time- and dose-dependent manner. When HT-29 cells were incubated with 80 or $160 \mathrm{mg} / \mathrm{l} \mathrm{COE}$ for $24 \mathrm{~h}$, autophagy and apoptosis were significantly induced (Fig. 2A and B).

Autophagy is normally considered to be a cell survival mechanism induced by stress, starvation and other environmental cues, but recent reports have provided evidence of cell death mediated by autophagy $(13,15)$. Numerous tumor suppressors, including death-associated protein kinase, phosphatase and tensin homolog and p53, can effectively stimulate autophagy in cancer cells (26). A previous study demonstrated that knocking down autophagy-related (Atg) 7, an important gene involved in autophagy, leads to uncontrolled proliferation of liver cancer cells beyond a normal lifespan in mice (35). Autophagy is now considered to be one of the most important types of PCD (type II PCD) (32). It begins with sequestering cytoplasmic organelles in a double-membrane vacuole called autophagosome $(13,14)$. Autophagosomes then fuse with lysosomes to form autolysosomes, where the internal materials are degraded and recycled (17). Detection of the autophagosome and autolysosomes inside cells is the best way to confirm induction of autophagy $(17,19)$. In the present study, observation of HT-29 cells by electron microscopy revealed that more autophagosomes and autolysosomes were present in cells treated with 80 or $160 \mathrm{mg} / \mathrm{l} \mathrm{COE}$ for $24 \mathrm{~h} \mathrm{COE}$ than in cells treated with $0,20,40 \mathrm{mg} / \mathrm{l} \mathrm{COE}$ for $24 \mathrm{~h}$ (Fig. 2A). LC3, a mammalian homolog of yeast Atg8, is another reliable marker of autophagosomes. During autophagy, the cytoplasmic form of LC3 (LC3-I, $18 \mathrm{kDa}$ ) is processed and recruited to autophagosomes, where the LC3-II form (16 kDa) is generated by site-specific proteolysis and lipidation near the C-terminus of LC3-I $(19,20)$. In the present study, immunoblotting of LC3 revealed a significant conversion of LC3-I to the autophagosomal membrane-bound LC3-II upon treatment with 80 or $160 \mathrm{mg} / \mathrm{l} \mathrm{COE}$ for $24 \mathrm{~h}$ (Fig. 2E and G). Beclin 1 is part of the class III PI3K lipid kinase complex, and plays a central role in the induction of autophagy (33). Immunoblot analysis demonstrated that the expression of Beclin 1 increased in COE-treated cells (Fig. 2E and G), suggesting that COE-induced autophagy was associated with augmented Beclin 1 expression. Taken together, these findings confirmed the induction of autophagy in HT-29 cells treated with COE.

It is well established that autophagy, mainly a self-digestion process, degrades intracellular structures in response to stress, leading to either cell survival or cell death $(15,16)$. To determine whether COE-induced autophagy increased cell survival or cell death, HT-29 cells were treated with $80 \mathrm{mg} / \mathrm{l} \mathrm{COE}$ for $24 \mathrm{~h}$ in the presence of 3-MA, a pharmacologic autophagy inhibitor that inhibits class III PI3K, which is known to inhibit autophagic sequestration $(13,22)$. The results revealed that treatment with 3-MA reduced the effect of COE on cell viability (Fig. 3D) and AVO formation (Fig. 3A). Furthermore, immunoblot analysis demonstrated that the expression of LC3-II was downregulated in cells treated with $80 \mathrm{mg} / \mathrm{l}$ $\mathrm{COE}$ for $24 \mathrm{~h}$ in the presence of 3-MA compared with that in cells treated with COE alone (Fig. 3C). By contrast, AVO formation increased upon treatment with $80 \mathrm{mg} / \mathrm{l} \mathrm{COE}$ in the presence of rapamycin in HT-29 cells (Fig. 3A), and the expression of LC3-II was upregulated (Fig. 3C). Of particular significance is the marked reduction in cell viability observed when autophagy was induced by COE administration in the presence of rapamycin (Fig. 3D). This finding confirmed that COE-induced autophagy did not enhance survival pathways 
but instead increased cell death in human colorectal cancer cells.

Our experimental results also demonstrated that, in addition to activating autophagy, COE induced pro-apoptotic signaling pathways leading to cell death. Electron microscopy observation of the COE-treated HT-29 cells revealed chromatin condensation, nuclear collapse and apoptotic body formation in cells treated with 80 or $160 \mathrm{mg} / \mathrm{l} \mathrm{COE} \mathrm{(Fig.} \mathrm{2B).} \mathrm{Apoptotic} \mathrm{cell}$ death was also quantified by determining the percentage of early apoptotic-gated cells (annexin $\mathrm{V}^{+}$) and late apoptotic-gated cells (annexin $\mathrm{V}^{+} / \mathrm{PI}^{+}$). The results revealed a progressive increase in the percentage of apoptotic cells in a COE dose-dependent manner (Fig. 2C and D). Bcl-2 and Bax are the key proteins for controlling the release of cytochrome $c$ and other pro-apoptotic factors from mitochondria, which leads to caspase activation and apoptotic cell death (36). The present study demonstrated that COE significantly decreased the expression of $\mathrm{Bcl}-2$ but increased the expression of Bax (Fig. 2F and H). This finding indicated that $\mathrm{COE}$ induced apoptosis by regulating the expression of the Bcl-2 family of proteins.

To further understand the mechanism of apoptosis and autophagy induced by COE, the PI3K/Akt/mTOR pathway was explored, which is frequently deregulated in cancer and is important for tumorigenesis, since this pathway can modulate apoptosis and autophagy $(13,15)$. mTOR exists in two conserved protein complexes, mTORC complex (mTORC) 1 and mTORC2 $(21,36,37)$. mTORC1 acts as a nutrient sensor and has been described as the master regulator of autophagy (37). When Akt is hyper-activated, mTORC1 can phosphorylate and activate mTOR, with the consequent inhibition of autophagy and apoptosis $(38,39)$. In the present study, immunoblot analysis revealed a robust and sustained decrease in Akt-Thr308, mTOR-Ser2448 and p70S6K-Thr389 levels upon treatment with increasing doses of COE (Fig. 4), indicating that COE may inhibit the proliferation of HT-29 cells by mediating the phosphorylation of Akt and reducing the kinase activity of mTOR. The reduction in MTOR activity promotes autophagy in HT-29 cells.

In conclusion, COE-induced autophagy and apoptosis synergize to inhibit colorectal cancer growth. The present study provides novel evidence that $\mathrm{COE}$ may be a promising agent against human colorectal cancer, particularly in light of the fact that cancer cells can escape induction of apoptosis, resulting in drug resistance. The present study may aid in the development of new strategies for the formulation of effective therapeutic drugs that would selectively target cancer cells and induce autophagic cell death independently of the normal apoptotic pathway.

\section{Acknowledgements}

The present study was financially supported by grants from the National Natural Science Foundation of China (Beijing, China; grant nos. 81403232, 81274141 and 81450051) and the Natural Science Foundation of Jiangsu Province of China (Nanjing, China; grant nos. BK 2012686 and SBK 2014021480).

\section{References}

1. Guo YQ, Li X, Xu J, Li N, Meng DL and Wang JH: Sesquiterpene esters from the fruits of Celastrus orbiculatus. Chem Pharm Bull (Tokyo) 52: 1134-1136, 2004.
2. Jin HZ, Hwang BY, Kim HS, Lee JH, Kim YH and Lee JJ: Antiinflammatory constituents of Celastrus orbiculatus inhibit the NF-kappa B activation and NO production. J Nat Prod 65: 89-91, 2002.

3. Li GQ, Liu D, Zhang Y, Qian Y, Zhang H, Guo S, Sunagawa M, Hisamitsu $T$ and Liu Y: Celastrol inhibits lipopolysaccharide-stimulated rheumatoid fibroblast-like synoviocyte invasion through suppression of TLR4/NF- $\mathrm{BB}$-mediated matrix metalloproteinase-9 expression. PloS One 8: e68905, 2013.

4. Li GQ, Liu D, Zhang Y, Qian YY, Zhu YD, Guo SY, Sunagawa M, Hisamitsu T and Liu YQ: Anti-invasive effects of celastrol in hypoxia-induced fibroblast-like synoviocyte through suppressing of HIF-1 $\alpha / C X C R 4$ signaling pathway. Int Immunopharmacol 17: 1028-1036, 2013.

5. Spivey AC, Weston M and Woodhead S: Celastraceae sesquiterpenoids: Biological activity and synthesis. Chem Soc Rev 31: 43-59, 2002.

6. Kim SE, Kim YH, Lee JJ and Kim YC: A new sesquiterpene ester from Celastrus orbiculatus reversing multidrug resistance in cancer cells. J Nat Prod 61: 108-111, 1998.

7. Westerheide SD, Bosman JD, Mbadugha BN, Kawahara TL, Matsumoto G, Kim S, Gu W, Devlin JP, Silverman RB and Morimoto RI: Celastrols as inducers of the heat shock response and cytoprotection. J Biol Chem 279: 56053-56060, 2004.

8. Nam NH: Naturally occurring NF-kappaB inhibitors. Mini Rev Med Chem 6: 945-951, 2006.

9. Zhang H, Qian Y, Liu Y, Li G, Cui P, Zhu Y, Ma H, Ji X, Guo $\mathrm{S}$ and Tadashi H: Celastrus orbiculatus extract induces mitochondrial-mediated apoptosis in human hepatocellular carcinoma cells. J Tradit Chin Med 32: 621-626, 2012.

10. Qian YY, Zhang H, Hou Y, Yuan L, Li GQ, Guo SY, Hisamits T and Liu YQ: Celastrus orbiculatus extract inhibits tumor angiogenesis by targeting vascular endothelial growth factor signaling pathway and shows potent antitumor activity in hepatocarcinomas in vitro and in vivo. Chin J Integr Med 18: 752-760, 2012.

11. Zhu YD, Liu YQ, Qian YY, Zhang H, Li GQ and Yang L: Extracts of Celastrus orbiculatus exhibit anti-proliferative and anti-invasive effects on human gastric adenocarcinoma cells. Chin J Integr Med, 2014 (Epub ahead of print).

12. Zhu Y, Liu Y, Qian Y, Dai X, Yang L, Chen J, Guo S and Hisamitsu T: Antimetastatic effects of Celastrus orbiculatus on human gastric adenocarcinoma by inhibiting epithelial-mesenchymal transition and NF- $\kappa \mathrm{B} /$ snail signaling pathway. Integr Cancer Ther 14: 271-281, 2015.

13. Lefranc F, Facchini V and Kiss R: Proautophagic drugs: A novel means to combat apoptosis-resistant cancers, with a special emphasis on glioblastomas. Oncologist 12: 1395-1403, 2007.

14. Levine B and Klionsky DJ: Development by self-digestion: Molecular mechanisms and biological functions of autophagy. Dev Cell 6: 463-477, 2004.

15. Levine B and Kroemer G: Autophagy in the pathogenesis of disease. Cell 132: 27-42, 2008.

16. Mizushima N, Levine B, Cuervo AM and Klionsky DJ: Autophagy fights disease through cellular self-digestion. Nature 451: 1069-1075, 2008.

17. Li J, Hou N, Faried A, Tsutsumi S and Kuwano H: Inhibition of autophagy augments 5-fluorouracil chemotherapy in human colon cancer in vitro and in vivo model. Eur J Cancer 46: 1900-1909, 2010.

18. Sharma N, Thomas S, Golden EB, Hofman FM, Chen TC, Petasis NA, Schönthal AH and Louie SG: Inhibition of autophagy and induction of breast cancer cell death by mefloquine, an antimalarial agent. Cancer Lett 326: 143-154, 2012.

19. Xi G, Hu X, Wu B, Jiang H, Young CY, Pang Y and Yuan H: Autophagy inhibition promotes paclitaxel-induced apoptosis in cancer cells. Cancer Lett 307: 141-148, 2011.

20. Pan X, Zhang X, Sun H, Zhang J, Yan M and Zhang H: Autophagy inhibition promotes 5-fluorouraci-induced apoptosis by stimulating ROS formation in human non-small cell lung cancer A549 cells. PloS One 8: e56679, 2013.

21. Ravikumar B, Vacher C, Berger Z, Davies JE, Luo S, Oroz LG, Scaravilli F, Easton DF, Duden R, O'Kane CJ and Rubinsztein DC: Inhibition of mTOR induces autophagy and reduces toxicity of polyglutamine expansions in fly and mouse models of Huntington disease. Nat Genet 36: 585-595, 2004.

22. Cui Q, Tashiro S, Onodera S, Minami M and Ikejima T: Autophagy preceded apoptosis in oridonin-treated human breast cancer MCF-7 cells. Biol Pharm Bull 30: 859-864, 2007.

23. Iwamaru A, Kondo Y, Iwado E, Aoki H, Fujiwara K, Yokoyama T, Mills GB and Kondo S: Silencing mammalian target of rapamycin signaling by small interfering RNA enhances rapamycin-induced autophagy in malignant glioma cells. Oncogene 26: 1840-1851, 2007. 
24. Maiuri MC, Le Toumelin G, Criollo A, Rain JC, Gautier F, Juin P, Tasdemir E, Pierron G, Troulinaki K, Tavernarakis N, et al: Functional and physical interaction between $\mathrm{Bcl}-\mathrm{X}(\mathrm{L})$ and a BH3-like domain in Beclin-1. EMBO J 26: 2527-2539, 2007.

25. Shintani T and Klionsky DJ: Autophagy in health and disease: A double-edged sword. Science 306: 990-995, 2004.

26. Levine B and Yuan J: Autophagy in cell death: An innocent convict? J Clin Invest 115: 2679-2688, 2005.

27. Kenific CM, Thorburn A and Debnath J: Autophagy and metastasis: Another double-edged sword. Curr Opin Cell Biol 22: 241-245, 2010.

28. Lock R and Debnath J: Extracellular matrix regulation of autophagy. Curr Opin Cell Biol 20: 583-588, 2008.

29. Li JJ, Yang J, Lu F, et al: Chemical constituents from the stems of Celastrus Orbiculatus. Chin J Nat Med 10: 279-283, 2012.

30. Zan K, Chen X-Q, Wang Q, et al: Chemical constituents in stem of Celastrus orbiculatus. Chin Trad Herbal Drugs 38: 1455, 2007.

31. Livak KJ and Schmittgen TD: Analysis of relative gene expression data using real-time quantitative PCR and the 2(-Delta Delta C(T)) method. Methods 25: 402-408, 2001.

32. Tait SW, Ichim G and Green DR: Die another way-non-apoptotic mechanisms of cell death. J Cell Sci 127: 2135-2144, 2014.
33. Levine B, Sinha S and Kroemer G: Bcl-2 family members: Dual regulators of apoptosis and autophagy. Autophagy 4: 600-606, 2008.

34. Ferlay J, Autier P, Boniol M, Heanue M, Colombet M and Boyle P: Estimates of the cancer incidence and mortality in Europe in 2006. Ann Oncol 18: 581-592, 2007.

35. Ramakrishnan S, Nguyen TM, Subramanian IV and Kelekar A: Autophagy and angiogenesis inhibition. Autophagy 3: 512-515, 2007.

36. Thomas S, Quinn BA, Das SK, Dash R, Emdad L, Dasgupta S, Wang XY, Dent P, Reed JC, Pellecchia M, et al: Targeting the Bcl-2 family for cancer therapy. Expert Opin Ther Targets 17: 61-75, 2013

37. Jung CH, Ro SH, Cao J, Otto NM and Kim DH: mTOR regulation of autophagy. FEBS Lett 584: 1287-1295, 2010.

38. Maiso P, Liu Y, Morgan B, Azab AK, Ren P, Martin MB, Zhang Y, Liu Y, Sacco A, Ngo H, et al: Defining the role of TORC1/2 in multiple myeloma. Blood 118: 6860-6870, 2011.

39. Corradetti MN and Guan KL: Upstream of the mammalian target of rapamycin: Do all roads pass through mTOR? Oncogene 25: 6347-6360, 2006. 